\title{
Perfil das Alunas no Departamento de Computação da Universidade de Brasília
}

\author{
Maristela Holanda, Marília Dantas, Gustavo Couto, Jan Mendonça Correa, \\ Aleteia Patrícia F. de Araújo, Maria Emília T. Walter \\ Departamento de Ciência da Computação - Universidade de Brasília (UnB) \\ Campus Darcy Ribeiro - CEP 70910-900 - Brasília - DF - Brasil \\ \{mholanda, jancorrea, aleteia, mariaemilia\}@unb.br, \\ \{alves.marilia, gcc.gustavo\}@gmail.com
}

\begin{abstract}
This paper presents the profile of the students of the course of the Department of Computer Science of the University of Brasilia. The profile is composed by analysis of the difference between the students (girls and boys) of the courses of Computer Science, Computer Engineering and Degree in Computing on the questions: entrance of the students; Reason for school dropout and academic grades.

Resumo. Este artigo apresenta o perfil das alunas do curso do Departamento de Computação da Universidade de Brasília. O perfil é composto por análise da diferença entre os alunos e alunas dos cursos de Ciência da Computação, Engenharia da Computação e Licenciatura em Computação nos quesitos: ingressantes nos cursos; motivo de desligamento e rendimento acadêmico.
\end{abstract}

\section{Introdução}

O Brasil tem em média apenas $17 \%$ de concluintes do sexo feminino em diferentes cursos de computação nos anos de 2000 a 2013, envolvendo Ciência da Computação, Engenharia da Computação, Sistema de Informação dentre outros (Maia, 2016). Essa realidade também pode ser observada em outros países (Keinan, 2017), (Vardi, 2015), (Stout, 2017).

Com o objetivo de diminuir esta disparidade, foi iniciado o projeto Meninas.comp: Computação também é coisa de menina!. Uma das ações deste projeto foi identificar o perfil das alunas nos cursos do Departamento da Computação da Universidade de Brasília (UnB). Este artigo apresenta uma análise dos dados acadêmicos das alunas nos cursos de Ciência da Computação, Engenharia da Computação e Licenciatura da Computação desta universidade.

Para isso, este artigo está dividido nas seguintes seções. Na Seção 2 são apresentados os dados dos ingressantes e desligados nos cursos do Departamento de Ciência da Computação da Universidade de Brasília. Na Seção 3 uma análise do desempenho acadêmico dos estudantes (alunos e alunas) desses cursos. E for fim, na Seção 4 as conclusões.

\section{Entrada e Desligamento nos Cursos de Computação na UnB}

Para esta investigação foram analisados dados relativos aos estudantes dos cursos do Departamento de Computação da UnB. A análise foi realizada a partir do ano de criação de cada curso, em 1987 foi a primeira turma do curso de Ciência da Computação, em 
1997 foi criado o curso de Licenciatura em Computação e somente em 2009 tem-se os primeiros ingressantes no curso de Engenharia da Computação. Os dados foram extraídos do Sistema de Informação Acadêmica de Graduação - SIGRA da UnB.

Figura 1 tem-se o número de ingressantes por ano e sexo no curso de Ciência da Computação, Licenciatura da Computação e Engenharia da Computação. As linhas em azul e cinza representam os alunos, e as cores amarela, vermelha e verde as alunas dos respectivos cursos. Como é possível observar, em todos os cursos de computação a entrada de meninos é bem maior que o de meninas. Uma questão importante é que a diferença entre o número de meninos e meninas que entraram nos cursos de computação aumentou com o tempo. No curso de Ciência da Computação, na sua primeira turma, o número de meninas (7) em relação ao número de meninos (15) foi de $46 \%$, nos anos 90 baixou para aproximadamente 30\%, e em 2013 ficou em 14\% o número de meninas (14) em relação aos meninos (98). Para o curso de Licenciatura em computação que iniciou no final da década de 90 , o número de entrada de meninas em relação aos meninos sempre foi menor que 20\%, chegando no ano de 2014 a apenas 6\%, 96 meninos e 6 meninas ingressantes no curso. Em Engenharia da Computação a maior porcentagem foi em 2010 com 23\% de meninas (15) em relação aos meninos (66), e a pior foi em 2015 com apenas 5\%, 4 meninas e 82 meninos.

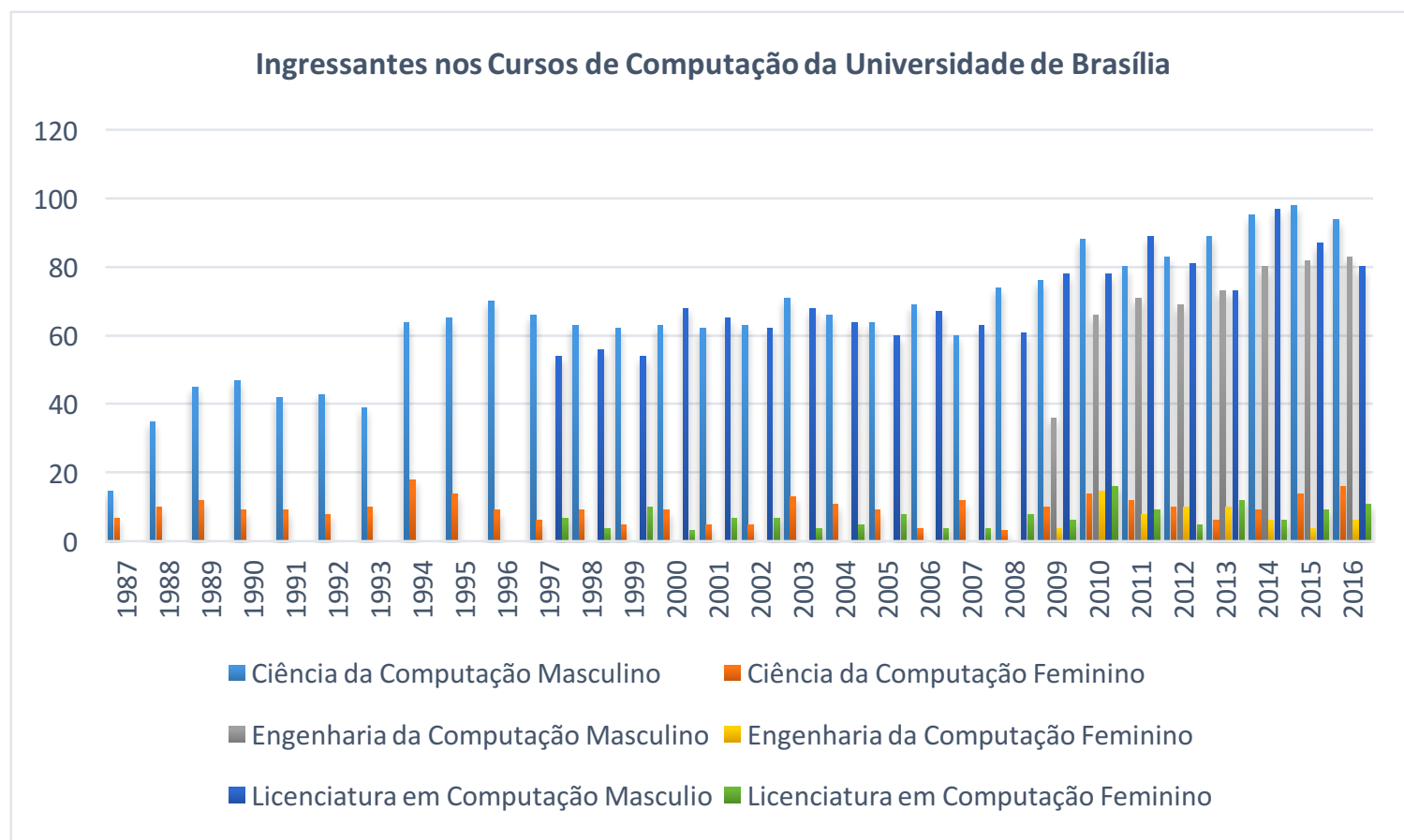

Figura 1. Ingressantes por sexo e ano nos cursos de Computação da UnB.

Um outro dado que fez parte da nossa análise está relacionado com o desligamento no curso (evasão), como pode ser observado na Figura 2. Para entendermos melhor o motivo que levaram os estudantes a se desligarem do curso, apresentamos na Figura 3 uma comparação entre meninas (lado esquerdo) e meninos (lado direito) do motivo que os levaram a serem desligados dos cursos de Ciência da Computação, Licenciatura em Computação e Engenharia da Computação. Os motivos de desligamento de estudantes em cursos na UnB envolvem as seguintes possibilidades: 
não cumpriu condição, o estudante tem um número mínimo de disciplinas que precisa ser aprovado ao longo de um ano, caso o estudante não cumpra esse requisito ele entra no estado de "Em condição", sendo necessário um plano de estudo para sair dessa condição, caso o aluno não cumpra esse plano de estudo ele é desligado da UnB; reprovação 3 vezes na mesma disciplina é outra regra da UnB que leva ao desligamento do estudante; transferência; desligamento voluntário pelo estudante; abandono do estudante; novo vestibular; e Outros.

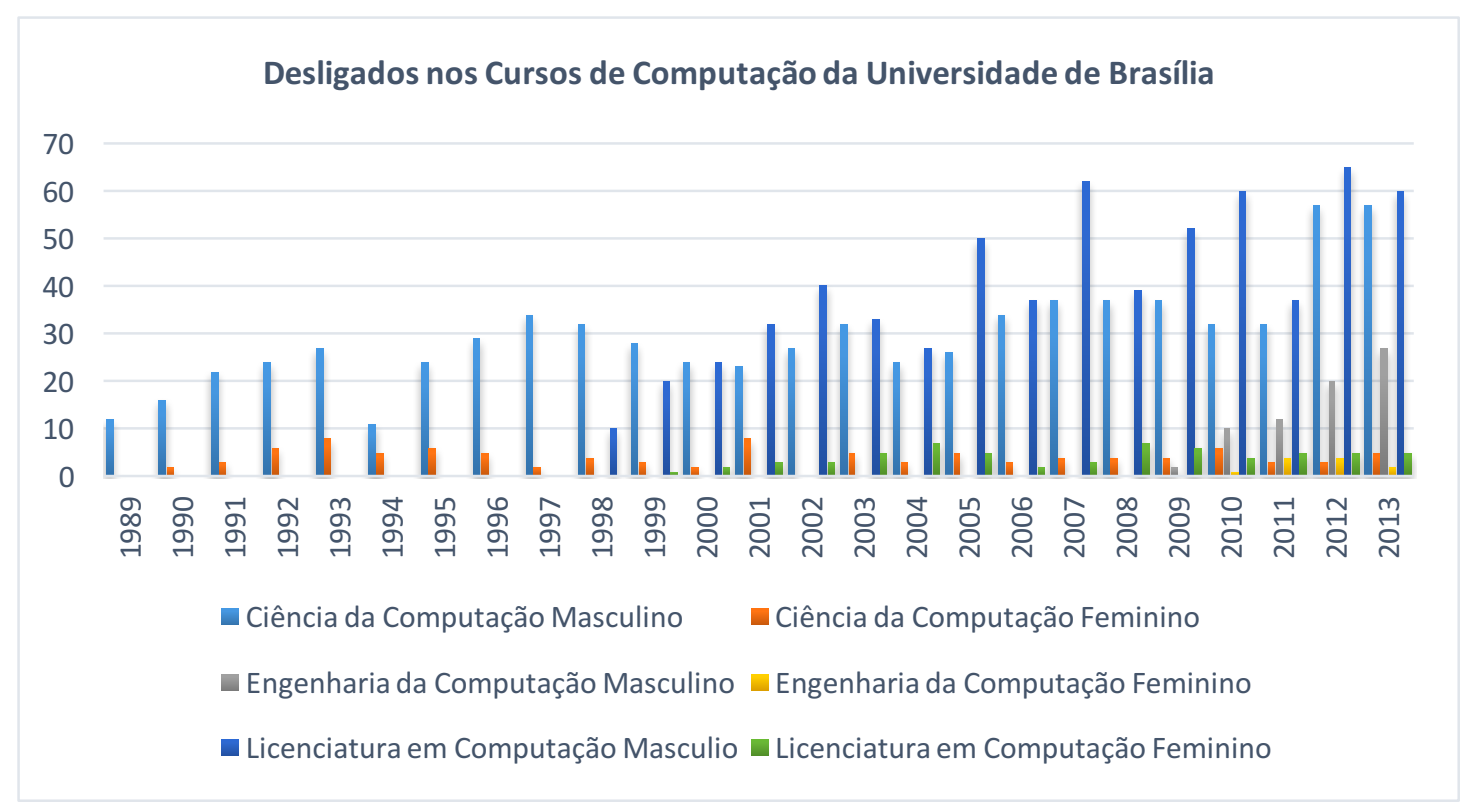

Figura 2. Desligados por sexo e ano nos cursos de Computação da UnB.

Na Figura 3 é possível observar que:

i) No curso de engenharia as alunas não foram desligadas por abandono, já $11 \%$ dos alunos foram desligados por este motivo;

ii) No curso de Ciência da Computação destacamos que as alunas não foram desligadas por reprovarem três vezes na mesma matéria, mas $6 \%$ dos alunos foram desligados por esse motivo;

iii) O desligamento por não cumprir condição é maior no curso de Licenciatura, principalmente entre as alunas;

iv) O desligamento voluntário é maior no curso de Ciência da Computação, chegando a ser o dobro em relação ao mesmo motivo nos outros cursos;

v) O desligamento por abandono no curso de Engenharia é muito inferior se comparado aos outros cursos;

vi) O desligamento por novo vestibular no curso de Engenharia é muito superior se comparado aos outros cursos;

vii) Por fim, o desligamento por transferência para outro curso praticamente existe somente no curso de Ciência da Computação. 

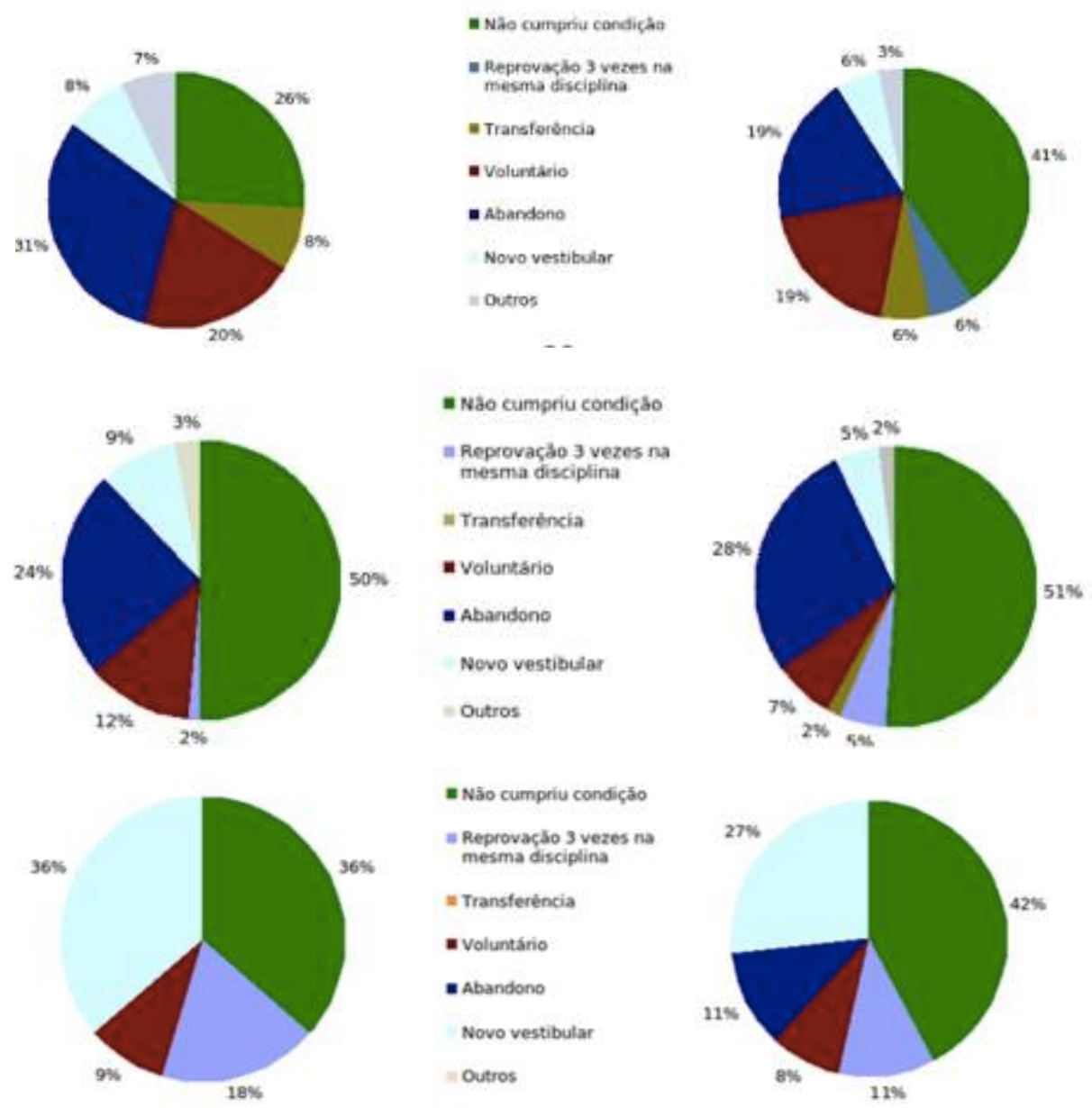

Figura 3. Motivo do desligamento, no lado esquerdo os dados das meninas, no direito os dos meninos na ordem Ciência da Computação, Licenciatura em Computação e Engenharia da Computação.

\section{Análise de Desempenho Acadêmico}

Os resultados apresentados foram calculados a partir do rendimento acadêmico dos alunos dos anos de 2000 até 2013. A Figura 4 apresenta os dados das médias das notas nas disciplinas dos cursos de computação nesse período, levando em consideração todas as disciplinas cursadas. Como pode ser observado as meninas tem uma ligeira melhora nas notas em relação aos meninos.

De acordo com a análise de dados realizada, identificou-se que os maiores índices de reprovação nos cursos de Computação da UnB eram as disciplinas: Física 1, Cálculo 1, Computação Básica (CB), Estrutura de Dados (ED), Organização de Arquitetura de Computadores (OAC), Software Básico e Organização de Arquivos (AO). A Figura 5 apresenta a média das notas dessas disciplinas, e como pode ser observado, é bem próxima a média de nota das meninas com a dos meninos, sendo que os meninos têm um ligeiro melhor desempenho na disciplina de $\mathrm{CB}$, a primeira disciplina de computação no curso, e as meninas tem uma média melhor de notas na disciplina de Software Básico. 


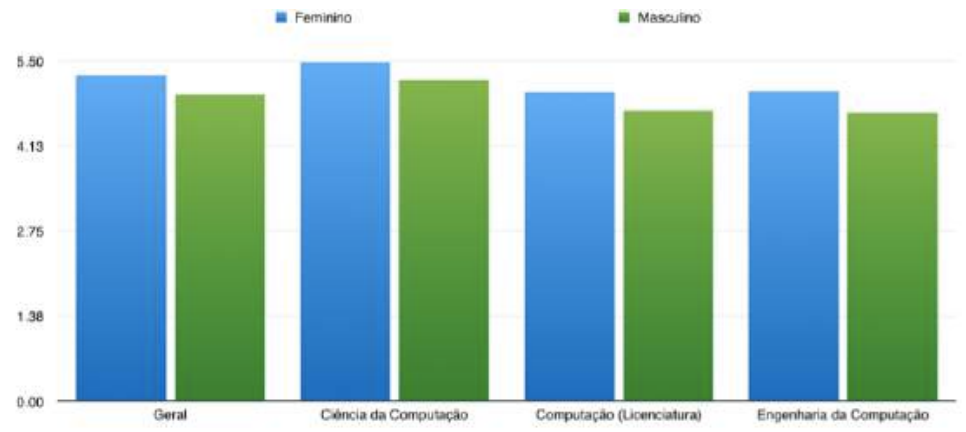

Figura 4. Desempenho acadêmico por sexo e cursos.

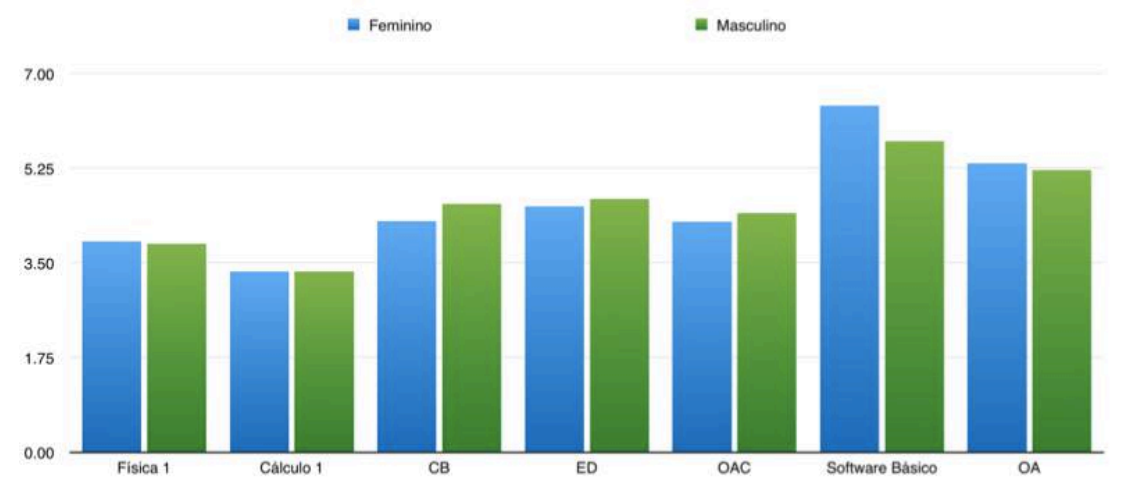

Figura 5. Desempenho acadêmico nas disciplinas com maior índice de reprovação por sexo.

\section{Conclusões}

O artigo apresenta um resumo da análise do perfil dos estudantes dos Cursos do Departamento de Ciência da Computação da UnB, analisando os três indicadores: ingressantes nos cursos; desligamentos dos alunos; e rendimento acadêmico. Identificamos a diminuição de ingressantes meninas em todos os cursos de computação da UnB. Em relação ao desligamento, os motivos de desligamento são bem variados em relação aos cursos. Em desempenho acadêmico, meninos e meninas tem média próxima de notas, porém olhando todas as disciplinas dos cursos as alunas têm, em média, um desempenho ligeiramente superior aos dos alunos.

\section{Referências}

Keinan, E.. (2017), "A New Frontier: But for Whom? An Analysis of the MicroComputer and Women's Declining Participation in Computer Science". CMC Senior Teses. 1466. Claremont Colleges..

Maia, M. M.. (2016), Limites de gênero e presença feminina nos cursos superiores brasileiros do campo da computação. Cadernos Pagu. n.46, pp. 223-244. ISSN 18094449.

Vardi, M. Y., (2015), What Can Be Done about Gender Diversity on Computing? A lot. n 58 (10). Communications of The ACM.

Stout, J. G., Grunberg, V. A., Ito, T. A. (2016), Gender Roles and Stereotypes about Science Careers Help Explain Women and Men's Science Pursuits. Sex Roles 75:490-499 DOI 10.1007/s11199-016-0647-5. 